Bora Özveren* and Selçuk Yücel
Department of Urology, Acibadem University School
of Medicine, Istanbul, Turkey
Dates: Received: 13 April, 2016; Accepted: 17 May,
2016; Published: 18 May, 2016
*Corresponding author: Bora Özveren, MD,
FEBU, Acibadem Kadikoy Hospital, Tekin Sok
No 8 Acibadem 34718, Istanbul, Turkey, Tel:
+90(216)5444276; +90(532)3475313; Fax:
+90(216)4284444; E-mail: ozverenb@yahoo.com;
bora.ozveren@acibadem.edu.tr
www.peertechz.com
ISSN: 2455-5495

\section{Case Report \\ Intractable Hematuria Due to Advanced Cardiac Failure and Venous Stasis}

\section{Introduction}

Intractable gross hematuria without an obvious or common urologic pathology needs more effort for correct diagnosis and appropriate management. In this case, we report on a patient with intractable hematuria originating from varicose vessels of urinary bladder due to advanced venous stasis after severe cardiac failure.

\section{Case Report}

A 66-year-old male was consulted for massive hematuria and clot retention subsequent to urethral catheterization after admission into intensive care unit because of cardiac decompensation. His medical history revealed cardiomyopathy and aortic metallic valve replacement 20 years ago and gout disease for 25 years. He had been on oral anticoagulation with warfarin for 20 years. On physical examination severe ascites and pulmonary effusion, and extensive venous stasis in lower trunk was found. Central venous pressure was $30 \mathrm{cmH} 2 \mathrm{O}$. His blood biochemistry work-up revealed moderate hyponatremia (132 meq/L) and a serum creatinin of $2.6 \mathrm{mg} / \mathrm{dl}$ (normal 0.5 to 1.4 ). Anticoagulation was terminated at first. Prothrombin time / INR level returned within normal limits following cessation of warfarin and he had only mild anemia.

Urologic ultrasonography was normal, a non-contrasted CT revealed cardiomegaly, bilateral pleural effusion, minimal hepatomegaly with mildly dilated intrahepatic veins, and thickened bladder wall. Diuretics and positive inotropic agents were used to restore cardiac function and to reduce the volume overload. Persistent blood loss required 1-2 units of transfusions daily and excessive clot retention necessitated frequent irrigations and re-catheterizations. The patient underwent cystoscopy where heavy bleeding was observed from largely dilated, fragile vessels at virtually everywhere in the bladder. Biopsies were taken to rule out any malignancy, vasculitis or amyloidosis. Pathology reported regular urothelium and edematous lamina propria. There were no findings of vasculitis or amyloid deposits. Studies for hematologic disorders and vasculitis were unrevealing. As his cardiac functions restored and volume overload was diminished by further medical management, the intensity of gross hematuria decreased. In a week, cardiac function deteriorated again and the severity of hematuria increased necessitating transfusions.
Consequent to the failure of conservative attempts at controlling hematuria, $2 \%$ formol was instilled into the bladder with gravity for 30 minutes under general anesthesia. In the early postoperative period, macroscopic hematuria ceased. Afterwards, his cardiac functions restored and he was dismissed on low-molecularweight heparin anticoagulation. However, two weeks later he was readmitted with pulmonary edema and massive hematuria requiring urethral catheterization with continuous irrigation. Following prompt management of volume overload, gross hematuria stopped. He was again discharged with anticoagulation but the patient died of cardiac failure two weeks later.

\section{Discussion}

Intractable hematuria originating from bladder may be related to many urologic diseases, other systemic diseases and adverse drug reactions. To our knowledge, severe cardiac failure has not been reported so far as a reason for intractable hematuria. Although one young male with myocardial infarction was reported to have hematuria, no detailed evaluation was carried out for the diagnostic and therapeutic management [1].

Even though hypervascularization of the bladder mucosa is a familiar image in bladder outlet obstructions, bladder infections and after endoscopic instrumentations, the findings of extensively enlarged, fragile vessels remain quite uncommon. Similar varicosities of bladder resulting in gross hematuria were associated with Klippel-Trenaunay syndrome, shistosomiasis, pregnancy, retroperitoneal fibrosis and telengiectasies due to cyclophospamide treatment [2-6]. In our case, there was no history of adverse drug reactions or findings of Klippel-Trenaunay syndrome that may result in hematuria, and the CT showed no sign of retroperitoneal fibrosis. In a way, the bleeding from the venous varicosities in bladder in this case may be considered similar to the condition due to the venous stasis and congestion that occurs in portal hypertension. In the absence of other underlying co-morbidities, congestive cardiac failure has plausibly contributed to the pathophysiology of the pelvic congestion and vesical varices in our case. 
When resistant to conservative measures, conjugated estrogens, hyperbaric oxygen therapy, endoscopic sclerotherapy, endoscopic coagulation, arterial embolization, formol or allum instillation and urinary diversion with or without cystectomy are viable alternatives for treatment [3]. When conservative methods failed, we only attempted formol instillation into the bladder. Although massive bleeding ceased, intermittent macroscopic hematuria persisted. Other alternatives of management could not be tried due to the high index of anesthesia risks and unrelenting cardiovascular conditions.

This case points out the potential risk of intractable hematuria related to severe venous engorgement in cardiac failure patients. As with several other systemic diseases with urological manifestations, conservative measures should be the initial step in controlling the hematuria since, as we experienced in this case, the proper management of the underlying cardiovascular pathology is entailed by resolution of hematuria.

\section{References}

1. Haywood LJ, Khan AH, Bornheimer J, Finck E, Tatter D (1997) Recurrent myocardial infarction with patent coronary arteries. J Natl Med A 89: 415-430.

2. Shekarriz B, Upadhyay J, Smith C, Kazmers A, Frontera R (2000) Massive hematuria in adults with Klippel-Trenaunay syndrome associated with vascular malformation of the bladder. Urol Int 64: 226-228.

3. Rodriguez Luna JM, Teruel JL, Vallejo J, Burgos FJ, Lovaca F, et al. (1992) Control of massive hematuria in idiopathic hemorrhagic cystitis after administration of conjugated estrogen. J Urol 148: 1524-1525.

4. Sano K, Shuin T, Takebayashi S, Sugawara T, Moriyama M, et al. (1989) A case of vesical varices as a complication of portal hypertension and manifested gross hematuria. J Urol 141: 369-371.

5. Hallamore SL, Grills RJ, Neerhut G, Lawrentschuk N (2007) Submucosal vesical varicosities causing hematuria and retention of urine in pregnancy: cystovarix. Am J Obstet Gynaecol 196: 29-30.

6. Bawany FA, Ghirano RA, Bayabani SR (2009) Primary vesical varices: a cause of gross hematuria. J Pak Med Assoc 59: 332-324.

Copyright: () 2016 Özveren B, et al. This is an open-access article distributed under the terms of the Creative Commons Attribution License, which permits unrestricted use, distribution, and reproduction in any medium, provided the original author and source are credited. 